\title{
Active synchronization in nonhyperbolic hyperchaotic systems
}

\author{
Elbert E. N. Macau, ${ }^{1}$ Celso Grebogi, ${ }^{2}$ and Ying-Cheng Lai ${ }^{3}$ \\ ${ }^{1}$ Brazilian National Institute for Space Research (INPE), São José dos Campos, São Paulo, 12227-010, Brazil \\ ${ }^{2}$ Instituto de Física, Universidade de São Paulo, Caixa Postal 66318, 05315-970 São Paulo, São Paulo, Brazil \\ ${ }^{3}$ Departments of Mathematics, Electrical Engineering, and Physics, Systems Science and Engineering Research Center, \\ Arizona State University, Tempe, Arizona 85287-1804 \\ (Received 17 April 2001; published 22 January 2002)
}

\begin{abstract}
We propose a methodology to address the outstanding problem of synchronization in nonhyperbolic hyperchaotic physical systems. Our approach makes use of a controlling-chaos strategy that accomplishes the task by transmitting only one scalar signal even in the presence of noise.
\end{abstract}

DOI: 10.1103/PhysRevE.65.027202

PACS number(s): 05.45.Xt, 05.45.Gg, 05.45.Vx

The inherent sensitive dependence on initial conditions implies that two trajectories starting from slightly different initial conditions diverge exponentially in time on the average. Despite that, it has been known that two chaotic systems can be synchronized [1]. Later on, Pecora and Carrol [2] gave a condition for the synchronization of two nearly identical chaotic systems: Using appropriately chosen state variables of a chaotic system (the driver) as input to a replica of the original system, the replica subsystem (the slave) might synchronize with the original system if its Lyapunov exponents are all negative. Since that work, synchronization in chaotic systems has become an area of intense activity [4,3].

In this report, we address the important problem of synchronization of nonhyperbolic hyperchaotic systems (systems with more than one positive Lyapunov exponent). Despite the success of a previous method [5] for synchronizing certain hyperchaotic systems, so far, to our knowledge, the physically relevant issue of nonhyperbolicity, which is typically represented by unstable dimension variability and can be extremely severe from the standpoint of shadowing $[6,7]$, has not been addressed. We present a general approach, based on the idea of controlling chaos [8], to synchronize nonhyperbolic chaotic systems in high dimensions by utilizing only one scalar transmitted signal. In particular, we apply small perturbations to some parameter of the slave system to synchronize it with the driver system. In the slave system, the current state of the driver system is obtained from the scalar transmitted signal by using the extended Kalman filter [9]. We call this approach the active synchronization to emphasize its main difference in relation to the more traditional passive synchronization process in which synchronization happens as a consequence of a proper coupling scheme [1-5]. To show that our ideas make sense, we use the pole-placement method [10] conveniently adapted to achieve robust active synchronization for the situations of strongly nonhyperbolic chaotic systems with more than one positive Lyapunov exponents and in the presence of noise. We mention that the concept of utilizing the principle of controlling chaos to achieve synchronization is, in fact, not new. It was suggested in Ref. [11]. However, the strategy proposed there is applicable to low-dimensional systems only and requires the transmission of all the state variables of the system from the driver to the slave. In contrast, the strategy proposed in this report allows for the synchronization of high-dimensional, nonhyperbolic chaotic systems with the transmission of a single scalar signal.

Intuitively, to achieve synchronization on hyperchaotic systems, the number of variables to be transmitted should be equal to that of positive Lyapunov exponents in order to account for the same number of unstable directions along the chaotic trajectory [12]. It was shown in Ref. [5] and in subsequent works introducing various improvements $[3,13,14]$ that, this belief is incorrect. In general, all those proposed approaches use feedback strategies whose parameters are fixed and are calculated using empirical strategies or optimization algorithms. As a consequence, none of those strategies can be considered to work for sure with any hyperchaotic system.

In all the ideas previously discussed, the requirement of a hyperbolic structure for the systems to be synchronized is implicit [15]. By its turn, nonhyperbolic systems can be classified into two types. For the first type, the splitting of the phase space into expanding and contracting subspaces is invariant along a trajectory except at the tangencies of the stable and unstable manifolds, where the angles between subspaces are zero [16]. The second type of nonhyperbolicity in hyperchaotic systems is due to unstable dimension variability [7]. It is related to the presence of unstable periodic orbits with different numbers of unstable directions embedded within the chaotic attractor. As a consequence, a typical trajectory experiences different numbers of unstable and stable directions as it evolves. Thus, the continuous splitting of the phase space into expanding and contracting subspaces is no longer valid.

Because of the global sensitivity, synchronization of chaotic systems having unstable dimension variability in the presence of noise or even small parameter mismatches is extremely difficult, if not impossible, to achieve. As synchronized trajectories move from one neighborhood to another having unstable periodic orbits with different number of expanding directions, they tend to separate exponentially from each other. What makes the situation hard for this type of nonhyperbolicity is the fact that the sets of periodic orbits with a different number of expanding directions are densely mixed [7]. Thus, regions where synchronization is highly susceptible to being destroyed due to the presence of noise extend over most of the attractor. As a result, previous methods $[5,14]$ cannot be expected to work for hyperchaotic sys- 


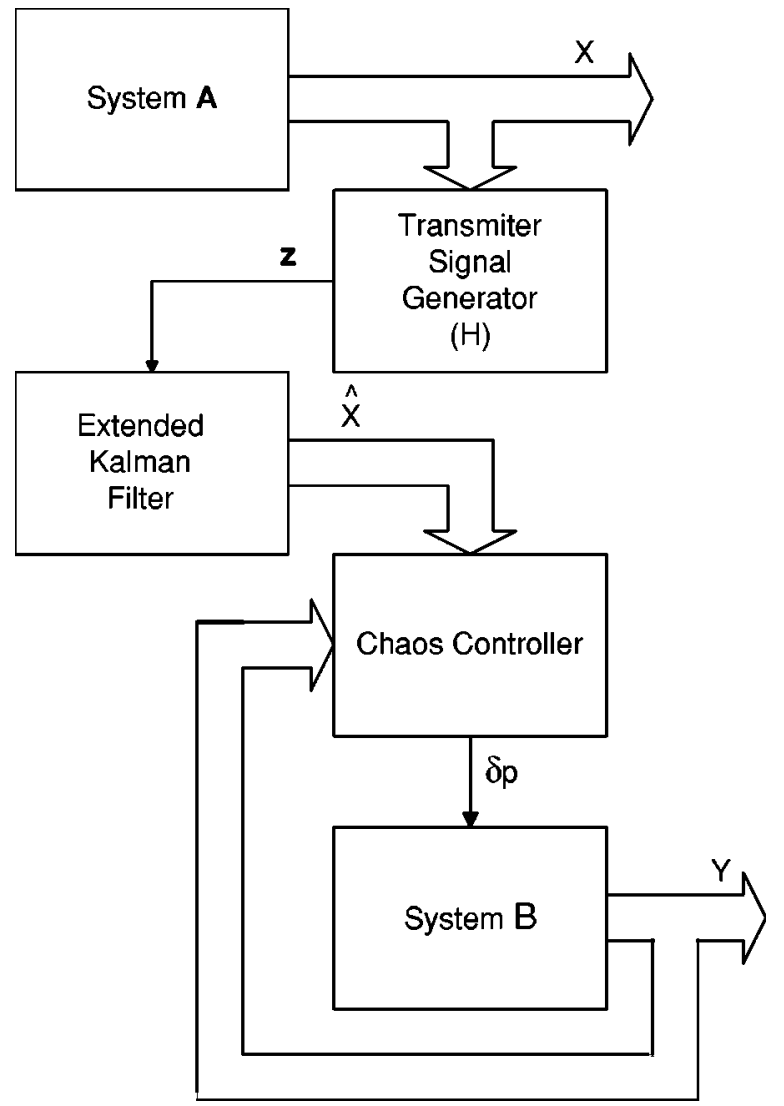

FIG. 1. Schematic illustration of our strategy of active synchronization.

tems with unstable dimension variability, especially for experimental implementation where noise is always present. In general, the densely mixed sets of periodic orbits with different numbers of expanding directions prevent the success of any passive feedback strategies of synchronization in which parameters of the systems are fixed.

A synchronization strategy will work for nonhyperbolic hyperchaotic systems only if it is capable of continuously keeping track of the local changes of the system as the trajectory evolves through the sets of periodic orbits with different numbers of expanding directions and changes its parameters accordingly. Thus, the main point here is to have a synchronization procedure with a built-in adjustment mechanism which monitors the system local dynamics and adjust its coefficients to keep the systems synchronized. In the remainder of this report, we detail our control strategy that implements exactly this principle.

A schematic illustration of our method to actively synchronize two chaotic systems is shown in Fig. 1. We extend the pole placement control of chaos strategy [10] to stabilize a chaotic trajectory of one system (System $B$ ) about a chaotic orbit of the other system (System $A$ ) to achieve synchronization. We assume that some parameter of the system can be externally adjusted, and that we have complete access to the state variables of the slave system (System $B)$. The extended Kalman filter allows us to estimate the current state of the driver system with the transmitted scalar signal. For concreteness, we consider two almost identical chaotic systems described by the following maps on the Poincare surface of section, $A: \mathbf{Y}_{i+1}=\mathbf{F}\left(\mathbf{Y}_{i}, \bar{p}\right)$ and $B: \mathbf{X}_{i+1}=\mathbf{F}\left(\mathbf{X}_{i}, p\right)$, where $\mathbf{X}_{i}, \mathbf{Y}_{i} \in \mathfrak{R}^{n}, \mathbf{F}$ is a smooth function in its variables, $\bar{p}$ for system $A$ is a fixed parameter value, and $p$ for system $B$ is an externally adjustable parameter whose value is restricted to lie in some small interval: $|p-\bar{p}|<\delta_{l m}$, about $\bar{p}$, where $\delta$ is a small number defining the range of parameter variation. Suppose that the two systems start with different initial conditions. The resulting chaotic trajectories are completely uncorrelated. Due to ergodicity, the two trajectories can get arbitrarily close to each other at a later time, say $i$. The difference between the trajectories in the next iteration is: $\mathbf{X}_{i+1}-\mathbf{Y}_{i+1}=\mathbf{F}\left(\mathbf{X}_{i}, p\right)-\mathbf{F}\left(\mathbf{Y}_{i}, \bar{p}\right)$. For values of $p$ close to $\bar{p}$, and as $\mathbf{X}_{i}$ falls in a small neighborhood of $\mathbf{Y}_{i}$, this equation can be linearily approximated in the neighborhood of $\mathbf{Y}_{i}$, as follows:

$$
\mathbf{X}_{i+1}-\mathbf{Y}_{i+1}^{\bar{p}}=\mathbf{A}_{i}\left[\mathbf{X}_{i}-\mathbf{Y}_{i}^{\bar{p}}\right]+\mathbf{B}_{i}\left(p_{i}-\bar{p}\right),
$$

where $\mathbf{A}_{i}$ is an $n \times n$ Jacobian matrix and $\mathbf{B}_{i}$ is an $n$-dimensional column vector: $\mathbf{A}_{i}=\left.\mathbf{D}_{\mathbf{Z}} \mathbf{F}(\mathbf{Z}, p)\right|_{\mathbf{Z}=\mathbf{Y}_{i}^{\bar{p}}, p=\bar{p}}$ and $\mathbf{B}_{i}=\left.\mathbf{D}_{p} \mathbf{F}(\mathbf{Z}, p)\right|_{\mathbf{Z}=\mathbf{Y}_{i}^{\bar{p}}, p=\bar{p}}$. Because of unstable dimension variability, the numbers of stable and unstable directions of the tangent space at each point of the trajectory change as the trajectory wanders on the chaotic invariant set. Let $s$ be the smaller of the number of stable directions for points of the set. Thus, a trajectory point of $A$ has at least $s$ stable directions in its tangent bundle. We arbitrarily choose $s$ vectors $\left\{\mathbf{v}_{i+u, 1}, \mathbf{v}_{i+u, 2}, \ldots, \mathbf{v}_{i+u, s}\right\}$ which span a subspace of dimension $s$ of the linearized stable manifold at the point $\mathbf{Y}_{i+u}^{\bar{p}}$. We define the matrix: $\Phi_{i, j}=\mathbf{A}_{i+u-1} \mathbf{A}_{i+u-2} \cdots \mathbf{A}_{i+j+1} \mathbf{A}_{i+j}$ for $j=1,2, \ldots,(u-1)$. To derive the control action to be applied to the parameter $p$ of system $B$ at each iteration, we iterate Eq. (1) $u$ times to obtain: $\mathbf{X}_{i+u}-\mathbf{Y}_{i+u}^{\bar{p}}$ $=\Phi_{i, 0}\left[\mathbf{X}_{i}-\mathbf{Y}_{i}^{\bar{p}}\right]+\Phi_{i, 1} \mathbf{B}_{i}\left(p_{i}-\bar{p}\right)+\Phi_{i, 2} \mathbf{B}_{i+1}\left(p_{i+1}-\bar{p}\right)+\cdots$

$+\mathbf{B}_{i+u-1}\left(p_{i+u-1}-\bar{p}\right)$. To make system $B$ synchronize with system $A, \mathbf{X}_{i+u}$ must land on the subspace of dimension $s$ of the linearized stable manifold of system $A$ 's trajectory at the point $\mathbf{Y}_{i+u}^{\bar{p}}$. Thus, parameter $p$ must be chosen such that there exists $s$ coefficients $\alpha_{1}, \alpha_{2}, \ldots, \alpha_{s}$ such that, $\mathbf{X}_{i+u}$ $-\mathbf{Y}_{i+u}^{\bar{p}}=\alpha_{1} \mathbf{v}_{i+u, 1}+\alpha_{2} \mathbf{v}_{i+u, 2}+\cdots+\alpha_{s} \mathbf{v}_{i+u, s}$. As a result, we obtain a system of $u+s$ equations with $u+s$ unknown variables: $p_{i}, p_{i+1}, \ldots, p_{i+u-1}, \alpha_{1}, \alpha_{2}, \ldots, \alpha_{s}$. This system can be solved for $p_{i}: p_{i}-\bar{p}=-\mathbf{K}_{i}^{t}\left[\mathbf{X}_{i}-\mathbf{Y}_{i}^{\bar{p}}\right]$, where $\mathbf{K}_{i}^{t}$ $=\mathbf{L C}_{i}^{-1} \Phi_{i, 0}, \quad \mathbf{L}=[-100 \cdots 0], \quad$ and $\quad \mathbf{C}_{i}=\left[-\Phi_{i, 1} \mathbf{B}_{i}, \ldots\right.$, $\left.-\mathbf{B}_{i+u-1}, \mathbf{v}_{i+u, 1}, \ldots, \mathbf{v}_{i+u, s}\right]$. This results in the values of parameter $p$ that must be applied to system $B$ at each iteration for the synchronization of the two systems, i.e., $\left|\mathbf{X}_{j}-\mathbf{Y}_{j}^{\bar{p}}\right|$ $\rightarrow 0$ for $j>i$.

The state variables of system $A$ can be estimated by using the extended Kalman filter [9], as follows. Assume that the transmitted signal can be described by the following map: $z_{i}=H\left(\mathbf{X}_{i}, v_{i}\right)$, where the random variable $v_{i}$ represents a white noise in the communication channel with normal probability distribution. We define $\hat{\mathbf{X}}_{i}^{-}$to be a priori state estimate at step $i$ given the knowledge of the system prior to step $i$, and $\hat{\mathbf{X}}_{i}$ a posteriori state estimate at step $i$ given the trans- 

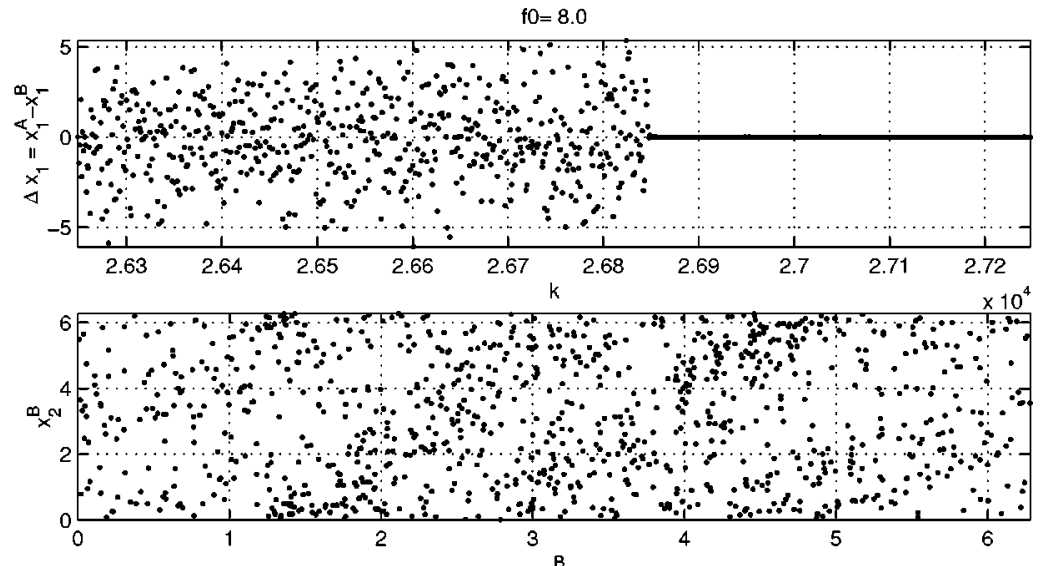

$x_{1}^{B}$

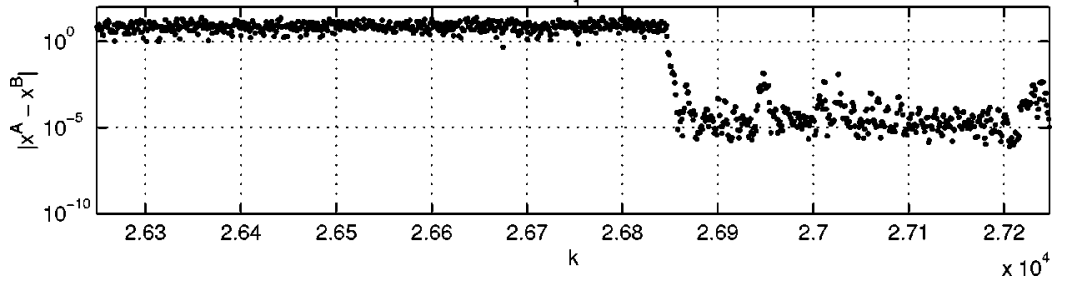

FIG. 2. Results of the use of our strategy to synchronize two double-rotor systems (System $A$ and System $B$ ) at $f=8.0$ (severe unstable dimension variability) when noise is present (see text for details): (a) time series of the difference between the $x_{1}$ phase variables of the systems, (b) Phase space evolution for $x_{1}$ and $x_{2}$ phase variables of System $B$; and (c) Time series of the euclidian distance between the phase variables of the systems. For graphics (a) and (c) $k$ stands for the number of iterations. The transmitted signal used is $x_{1}^{A}+x_{2}^{A}+x_{3}^{A}+x_{4}^{A}+v$, where $v$ is a white noise with normal probability distribution and signal-to-noise ratio equal to $70 d B$. Initially, the systems are far apart by an ecludian distance of one unit. mitted signal $z_{i}$. Our goal is to find an equation that computes an a posteriori state estimate $\hat{\mathbf{X}}_{i}$ as a combination of an a priori estimate $\hat{\mathbf{X}}_{i}^{-}$and a weighted difference between an actual measurement $z_{i}$ and a measurement prediction $H\left(\hat{\mathbf{X}}_{i}^{-}, 0\right)$, as follows: $\hat{\mathbf{X}}_{i}=\hat{\mathbf{X}}_{i}^{-}+\mathbf{K}_{i}\left[z_{i}-H\left(\hat{\mathbf{X}}_{i}^{-}, 0\right)\right]$, where $\hat{\mathbf{X}}_{i}^{-}=\mathbf{F}\left(\hat{\mathbf{X}}_{i-1}, \bar{p}\right)$. The gain $\mathbf{K}_{i}$ at each iteration, is calculated by using the following set of equations, which minimizes the a posteriori estimate error covariance

$$
\begin{gathered}
\mathbf{P}_{k}=E\left[\left(\mathbf{X}_{i}-\hat{\mathbf{X}}_{i}\right)\left(\mathbf{X}_{i}-\hat{\mathbf{X}}_{i}\right)^{T}\right]: \mathbf{P}_{i}^{-}=\mathbf{A}_{i-1} \mathbf{P}_{i-1} \mathbf{A}_{i-1}^{T}, \\
\mathbf{K}_{i}=\mathbf{P}_{i}^{-} \mathbf{C}_{i}^{T}\left(\mathbf{C}_{i} \mathbf{P}_{i}^{-} \mathbf{C}_{i}^{T}+\mathbf{D}_{i} R \mathbf{D}_{i}^{T}\right)^{-1},
\end{gathered}
$$

and $\mathbf{P}_{i}=\left(\mathbf{I}-\mathbf{K}_{i} \mathbf{C}_{i}\right) \mathbf{P}_{i}^{-}$, where $\mathbf{C}_{i}=\left.\mathbf{D}_{\mathbf{Z}} H(\mathbf{Z}, v)\right|_{\mathbf{Z}=\mathbf{x}_{i}, v=0}$ and $\mathbf{D}_{i}=\left.\mathbf{D}_{\mathbf{v}} H(\mathbf{Z}, v)\right|_{\mathbf{Z}=\mathbf{x}_{i}, v=0}$.

We now apply our ideas to a physical system: the double rotor map $[17,10]$, which is a four-dimensional map describing the time evolution of a mechanical system consisting of two connected massless rods. This system is modeled by the following four-dimensional map, which describes the dynamics of the rotor relating the state of the system immediately after consecutive kicks

$$
\begin{gathered}
\boldsymbol{\Theta}_{i+1}=\mathbf{M} \boldsymbol{\Phi}_{i}+\boldsymbol{\Theta}_{i}, \\
\boldsymbol{\Phi}_{i+1}=\mathbf{L} \boldsymbol{\Phi}_{i}+\mathbf{G}\left(\boldsymbol{\Theta}_{i+1}\right),
\end{gathered}
$$

where $\boldsymbol{\Theta}^{T}=[x(1), x(2)]^{T}, \boldsymbol{\Phi}^{T}=[x(3), x(4)]^{T}$, and $\mathbf{G}(\boldsymbol{\Theta})^{T}$ $=\left[c_{1} \sin x(1), c_{2} \sin x(2)\right]^{T},\left(\Theta \in S^{1} \times S^{1}\right.$, and $\left.\boldsymbol{\Phi} \in \operatorname{Re} \times \operatorname{Re}\right)$. In Eq. (2), $x(1,2)$ are the angular positions of the rotors at the instant of the $i$ th kick, while $x(3,4)$ are the angular velocities of the rotors, $\mathbf{L}$ and $\mathbf{M}$ are $2 \times 2$ constant matrices whose elements depend on the physical parameters of the rotors, $c_{1}$ and $c_{2}$ are two parameters that are proportional to the kicking strength $f$, which we choose to be the externally adjust- able control parameter. We choose parameters such that $c_{1}$ $=c_{2}=f$. For $\mathbf{L}$ and $\mathbf{M}$, we use the setting values from Ref. [10].

For the double-rotor map, numerical experiments [10] show that the system goes through a cascade of perioddoubling bifurcations for $f<f_{1} \simeq 6.75$ and becomes chaotic with one positive Lyapunov exponent at $f_{1}$. For values of $f$ near $f=8.0$, there is a transition from one positive Lyapunov exponent to two positive ones and, about this parameter value, it exhibits fluctuations between one and two positive exponents in any finite times. These fluctuations reflect the presence of unstable dimension variability for this system. For values of $f$ much above the transition point, as is the case for $f=9.0$, the second Lyapunov exponent becomes so positive that finite-time fluctuations of this exponent will only have a negligible tail on the negative side. Thus, for $f$ near 8.0 , the chaotic attractor of the double rotor map is nonhyperbolic with severe unstable dimension variability, while for $f$ about 9.0, the attractor has two positive Lyapunov exponents and exhibits essentially nonhyperbolic tangencies of the stable and the unstable manifolds. Figure 2 shows the results of applying our control strategy to synchronize two double-rotor systems for $f=8.0$. The transmitted signal used is $z_{i}=H\left(\mathbf{X}_{i}, v_{i}\right)=x(1)+x(2)+x(3)+x(4)+v$, where $v$ is a white noise with normal probability distribution and the signal-to-noise ratio is $70 \mathrm{~dB}$. This result does not change even if each individual parameter of the model for System B are changed up to $1 \%$. Thus, we observe that, even in the presence of noise or parameter mismatch, small control perturbations applied to $B$ result in robust synchronization [18]. For $f=9.0$, the results are similar. The remarkable result is that our active approach works equally well for nonhyperbolic hyperchaotic systems having unstable dimension variability or tangencies of stable and unstable manifolds. In contrast, for the nonhyperbolic case of $f=8.0$, the previous methodology $[5,14]$ fails, as we have tested. 
In summary, we have proposed a general active method for achieving synchronization in nonhyperbolic hyperchaotic physical systems. Our strategy employs a built-in adjustment mechanism that monitors the system's local dynamics and perturbs the system to maintain robust synchronization, regardless of whether the nonhyperbolic hyperchaotic systems exhibit unstable dimension variability or tangencies of mani- folds. We expect our methodology to be practically appealing for experimental implementation of chaos synchronism in high dimensions.

E.E.N.M. and C.G. were sponsored by CNPQ and by FAPESP. Y.C.L. was sponsored by AFOSR under Grant No. F49620-98-1-0400 and by NSF under Grant No. PHY9722156.
[1] H. Fujisaka and T. Yamada, Prog. Theor. Phys. 69, 32 (1983); V. S. Afraimovich, N. N. Verichev, and M. I. Rabinovich, Radiophys. Quantum Electron. 29, 747 (1986).

[2] L. M. Pecora and T. L. Carroll, Phys. Rev. Lett. 64, 821 (1990).

[3] L. M. Pecora, T. L. Carroll, G. A. Johnson, D. J. Mar, and J. F. Heagy, Chaos 7, 520 (1997).

[4] U. Parlitz, L. O. Chua, L. Kocarev, K. S. Halle, and A. Shang, Int. J. Bifurcation Chaos Appl. Sci. Eng. 2, 973 (1992); K. M. Cuomo and A. V. Oppenheim, Phys. Rev. Lett. 71, 65 (1992); 71, 65 (1992). K. M. Cuomo, A. V. Oppenheim, and S. H. Strogatz, Int. J. Bifurcation Chaos Appl. Sci. Eng. 3, 1629 (1993).

[5] J. H. Peng, E. J. Ding, M. Ding, and W. Yang, Phys. Rev. Lett. 76, 904 (1996).

[6] R. Abraham and S. Smale, Proc. Symp. Pure Math. 14, 5 (1970).

[7] S. P. Dawson, C. Grebogi, T. Sauer, and J. A. Yorke, Phys. Rev. Lett. 73, 1927 (1994); S. P. Dawson, ibid. 76, 4348 (1996); E. J. Kostelich, I. Kan, C. Grebogi, E. Ott, and J. A. Yorke, Physica D 109, 81 (1997); T. Sauer, C. Grebogi, and J. A. Yorke, Phys. Rev. Lett. 79, 59 (1997); Y.-C. Lai, C. Grebogi, and J. Kurths, Phys. Rev. E 59, 2907 (1999); Y.-C. Lai and C. Grebogi, Phys. Rev. Lett. 82, 4803 (1999).

[8] E. Ott, C. Grebogi, and J. A. Yorke, Phys. Rev. Lett. 64, 1196 (1990); C. Grebogi and Y.-C. Lai, Syst. Control Lett. 31, 407 (1997); S. Boccaletti, C. Grebogi, Y.-C. Lai, H. Mancini, and D. Maza, Phys. Rep. 329, 103 (2000).

[9] R. E. Kalman, Trans. ASME J. Basic Eng. 83, 35 (1960); R. G. Brown and P. Y. C. Hwang, Introduction to Random Signals and Applied Kalman Filtering, 3rd ed. (Wiley, New York, 1997).
[10] F. J. Romeiras, C. Grebogi, E. Ott, and W. P. Dayawansa, Physica D 58, 165 (1992).

[11] Y.-C. Lai and C. Grebogi, Phys. Rev. E 47, 2357 (1993).

[12] K. Pyragas, Phys. Lett. A 181, 203 (1993).

[13] L. Kocarev and U. Parlitz, Phys. Rev. Lett. 74, 5028 (1995).

[14] A. Tamasevicius and A. Cenys, Phys. Rev. E 55, 297 (1997); M. K. Ali and J. Q. Fang, ibid. 55, 5285 (1997); G. A. Johnson, D. J. Mar, T. L. Carroll, and L. M. Pecora, Phys. Rev. Lett. 80, 3956 (1998).

[15] D. K. Arrowsmith and C. M. Place, An Introduction to Dynamical Systems (Cambridge University, Cambridge, 1994).

[16] S. M. Hammel, J. A. Yorke, and C. Grebogi, J. Complexity 3, 136 (1987); C. Grebogi, S. M. Hammel, J. A. Yorke, and T. Sauer, Phys. Rev. Lett. 65, 1527 (1990); Y.-C. Lai, C. Grebogi, J. A. Yorke, and I. Kan, Nonlinearity 6, 779 (1993). 779 (1993).

[17] C. Grebogi, E. Kostelich, E. Ott, and J. A. Yorke, Physica D 25, 347 (1987).

[18] The issue of long transient time before synchronization can be achieved is inherent for high-dimensional chaotic systems, as the probability for two trajectories to come close is smaller in a higher-dimensional phase space. Theoretically, a feasible way to reduce the transient time is to use the "targeting" technique that utilizes the principle of control to drive two trajectories close in the shortest possible time. Existing works on targeting focus, however, exclusively on unstable periodic orbits [T. Shinrot, C. Grebogi, E. Ott, and J. A. Yorke, Phys. Rev. Lett. 65, 3215 (1990); E. J. Kostelich, C. Grebogi, E. Ott, and J. A. Yorke, Phys. Rev. E 47, 305 (1993); Y.-C. Lai, Phys. Lett. A 221, 375 (1996).] However, to target a chaotic trajectory in high-dimensional phase space in the presence of severe nonhyperbolicity remains an unsolved problem. 